\title{
émulations
}

\section{Pierre Rosanvallon - Le bon gouvernement}

Julie Alev Dilmaç

Émulations - Revue de sciences sociales

2018. Comptes rendus critiques. En ligne

\section{Article disponible à l'adresse suivante}

https://ojs.uclouvain.be/index.php/emulations/article/view/7503

\section{Pour citer cet article}

Julie Alev Dilmaç, «Pierre Rosanvallon - Le bon gouvernement », Émulations, en ligne. Mise en ligne le 29 novembre 2018.

DOI : 10.14428/emulations.cr.049

Distribution électronique : Université catholique de Louvain (Belgique) : ojs.uclouvain.be

(C) Cet article est mis à disposition selon les termes de la Licence Creative Commons Attribution, Pas d'Utilisation Commerciale 4.0 International. http://creativecommons.org/licenses/by-nc/4.0/

Éditeur : Émulations - Revue de sciences sociales / Presses universitaires de Louvain https://ojs.uclouvain.be/index.php/emulations

ISSN électronique : 1784-5734

UCL PRESSES

UNIVERSITAIRES

DE LOUVAIN 


\section{Pierre Rosanvallon - Le bon gouvernement}

Julie Alev Dilmaç

Recensé : Pierre Rosanvallon (2017), Le bon gouvernement, Paris, Seuil (« Les Livres du nouveau monde »), $416 \mathrm{p}$.

"Nos régimes peuvent être dits démocratiques, mais nous ne sommes pas gouvernés démocratiquement » (p. 9). Tel est le postulat que Pierre Rosanvallon tente de démontrer tout au long de son nouvel ouvrage, Le bon gouvernement. C'est dans un jargon des sciences politiques que l'auteur relate ici l'histoire de l'exercice des démocraties ; démocraties qui relèvent pour lui plus d'un mode de gouvernement que d'un régime. Malgré l'abondance d'illustrations contextualisées dans le temps et puisées dans diverses sociétés (et notamment en France, en Allemagne, aux États-Unis ou encore en Angleterre), le sujet abordé ici s'inscrit aisément dans le monde contemporain. Les qualités requises par les élus pour " bien " gouverner, les attentes des citoyens face à la démocratie, ainsi que les limitations et dérives dont ce type de gouvernement peut faire preuve sont les thèmes principaux de cet ouvrage.

En vue de construire son argumentaire, Rosanvallon a divisé son analyse en quatre chapitres principaux, dont le premier fait état des transformations majeures sur le plan juridique portées par la Révolution qui ont conduit au déclin de l'exécutif et à l'avènement de l'impersonnalité. Dans le deuxième chapitre, l'auteur se focalise sur le thème de la " présidentialisation » des démocraties en évoquant les deux expériences pionnières dans ce domaine, à savoir celle de 1848 en France et celle préconisée par la constitution de Weimar. Un autre sujet est par ailleurs longuement traité ici : il s'agit de la personnalisation du pouvoir. En prenant appui sur le régime institué en France par le général de Gaulle, l'auteur montre comment, peu à peu, « s'est instauré un écart qui n'a cessé de s'accroitre entre la forme politique de la présidentialisation-personnalisation et son incarnation sociale » (p. 153). On y apprend que le pouvoir se doit d'avoir un visage. Le troisième chapitre de cet ouvrage est dédié à la description du rapport entre gouvernants et gouvernés. L'auteur montre comment, selon les époques, les attentes des gouvernés vis-à-vis des gouvernants, mais aussi les attitudes de ces derniers envers les citoyens, ont évolué : l'idée principale qui s'en dégage est que gouverner pouvait être perçu comme un art du maniement de la force, tantôt basé sur la ruse ou la séduction, tantôt sur la dissimulation. Les citoyens, quant à eux, ont longtemps été appréhendés comme une masse, une "populace ", perçue comme soumise à des pulsions que seul un gouvernant fort pouvait dompter. Le concept de « distance » entre ces deux groupes est de même intéressant

\footnotetext{
${ }^{1}$ Maitre de conférences en Sociologie à la Cyprus International University et membre associé du Centre de Philosophie, d'Épistémologie et de Politique (PHILéPOL), Université Paris Descartes, Sorbonne Paris Cité.
} 
ici : on constate que, par le passé, les citoyens avaient tendance à apprécier les représentants politiques qui avaient fait preuve d'expériences antérieures, et si possible d'exploits; cette situation faisait de ces hommes politiques des individus quasi " extraordinaires " qu'il était difficile d'approcher, maintenant ainsi une certaine distance avec le peuple. Aujourd'hui, nous serions plus enclins à attendre d'un·e candidat.e qu'il ou elle soit " près du peuple " ou qu'il ou elle nous ressemble. Les caractéristiques de la figure du bon gouvernant sont d'ailleurs étayées dans le quatrième chapitre de l'ouvrage : l'auteur y décrit les principales vertus dont I'homme politique devrait être doté dans une démocratie qu'il qualifie de " confiance ".

Cet ouvrage propose des pistes en vue de rétablir l'équilibre entre les gouvernés et les gouvernants, équilibre que l'auteur considère comme fondamental à la pérennité démocratique. C'est à la lumière d'illustrations historiques et politiques que Rosanvallon entend appréhender la situation actuelle et nous éclairer sur ces temps politiquement troublés, caractérisés notamment par un défaut de représentation des citoyens de la part des hommes politiques.

L'analyse du mode démocratique et la place du peuple gouverné dans ce type de gouvernement font partie des sujets que l'auteur affectionne, puisque déjà traités dans plusieurs de ses ouvrages précédents : on se souviendra notamment du livre Le Sacre du citoyen (Rosanvallon, 1992) dans lequel il décrivait l'émergence de la figure du citoyen électeur, thème prolongé dans Le peuple introuvable (Rosanvallon, 1998) où il s'interrogeait sur la notion de " peuple » et sur la représentation déficiente des citoyens dans la démocratie moderne; ou encore de La Contre démocratie (Rosanvallon, 2006²) dans lequel Rosanvallon livrait, entre autres, une analyse des causes de la méfiance des citoyens face aux représentants du pouvoir : il y démontrait notamment que l'érosion de la confiance envers les dirigeants avait fait naitre une "société de la défiance ".

Bien que s'inscrivant dans le prolongement de ces études, c'est avec une autre problématique que l'auteur revient ici : les démocraties seraient sujettes à un phénomène redéfinissant leur mode de fonctionnement. Cette situation serait provoquée par la montée en puissance du pouvoir exécutif, devenu aujourd'hui le pivot des démocraties, et ce au détriment du pouvoir législatif. Ce basculement aurait fait naitre un modèle présidentiel-gouvernant (la « présidentialisation »), marquant depuis trois décennies une rupture dans la nature et la forme des démocraties : c'est de ce phénomène et de ses conséquences sur la relation entre gouvernés et gouvernants dont il est question dans cet ouvrage.

Selon le politiste, avec l'avènement de la présidentialisation, définie par l'élection au suffrage universel du chef de l'exécutif, les personnalités détentrices du pouvoir se seraient vues "normalisées": elles seraient dorénavant perçues comme les responsables directes des maux sociaux et des problèmes personnels des citoyens. Ce modèle de gouvernement a permis aux individus de mettre un "visage " sur les fonctionnements administratifs jusqu'ici trop opaques; ils sont donc plus enclins à vouloir interpeler les dirigeants sur leurs agissements et à souhaiter leur demander des comptes.

2 On pourra aussi se référer à la recension que j'ai consacrée à cet ouvrage (Dilmaç, 2007). 
Cependant, cette situation est loin de signifier que les citoyens soient plus écoutés ou même entendus par les gouvernants : selon l'auteur, le peuple votant se trouverait « bien peu souverain » (p. 9) puisque, passé le moment électoral, il ne prendrait plus part aux décisions.

L'élection alors, si elle est désignée comme la modalité phare des démocraties, n'est en aucun cas suffisante à elle seule pour définir ce mode de gouvernement, car « un pouvoir est [...] considéré comme démocratique que s'il est soumis à des épreuves de contrôle et de validation » (p. 32). Pour lutter contre la mal-représentation, diverses solutions ont d'ailleurs été proposées antérieurement : tirage au sort, limitation du cumul des mandats, démocratie participative ou même directe. Or aucune ne semble avoir comblé ce déficit de démocratie.

En outre, l'auteur démontre que le vote du représentant exécutif dans le présidentialisme se heurte à des dichotomies propres à toutes élections : élire un gouvernant ne signifie pas lui conférer toute légitimité ou même valider automatiquement toutes les actions ou les décisions prises par le pouvoir en place. De plus, comme le remarque Rosanvallon, l'élection relève d'une compétition dont le résultat n'exprime que l'opinion d'une partie de la majorité et non celle d'une volonté générale, comme il l'est souvent avancé. L'élection correspondrait alors à une procédure de sélection qui s'achève sur « une légitimation inaccomplie » (p. 159).

Une autre dichotomie mise en avant par l'auteur concerne les qualités des candidats à une élection : un « bon candidat " ne serait pas nécessairement " un bon gouvernant " puisque le premier s'affairerait à séduire les électeurs, en manifestant par exemple une certaine proximité feinte avec le peuple ; il tiendrait d'ailleurs plusieurs langages pour mener à bien son entreprise. Le second, quant à lui, se montrerait constant dans ses choix, suivrait la même direction quelles que soient les circonstances, et s'en tiendrait à ses dires. Cependant, comment conquérir l'opinion publique sans exercer l'art de la séduction et celui de la manipulation ? Faut-il être un élu " positivement quelconque " (d'autres diront « normal »), proche des citoyens ou, au contraire, un élu " expert » qui se démarque des gouvernés en mettant en exergue des attributs spécifiques associés à la fonction politique ? Bien que n'apportant pas de réponses précises à ces questionnements, Rosanvallon insiste sur le fait que « ce sont toujours les mêmes recettes, les mêmes subterfuges, les mêmes éléments de langage qui guident la conduite de gouvernants hantés par la conservation de leur pouvoir, l'avènement de l'âge médiatique puis électronique n'ayant fait que multiplier les instruments de manipulation » (p. 197).

Cette problématique relevant de la représentation en politique s'impose d'ailleurs comme un des fils conducteurs de l'ouvrage. Les démocraties contemporaines seraient dorénavant rongées par le mal-gouvernement, caractérisé par la mise à distance des citoyens. En effet, ceux-ci sont écartés du processus de prise de décision par les gouvernants une fois l'élection passée. Pour arriver à leur fin, diverses stratégies, notamment langagières, semblent être usitées par les élus : glissements sémantiques, approximations, profération de mensonges, utilisation de termes trop vagues ou excessivement jargonneux; I'homme politique opterait de même pour le monologue, ôtant au citoyen toute chance de réplique, le plongeant alors dans une écoute passive... Autant de processus qui amplifieraient l'écart entre gouvernés et gouvernants, car rendant « illisibles » les décisions politiques aux yeux des citoyens. Cette opacité 
remettrait d'ailleurs en question le principe de souveraineté du peuple, puisqu'elle empêcherait les citoyens d'exercer des fonctions démocratiques.

Les élus eux-mêmes ne se soucieraient pas de l'avis des gouvernés lors de leur prise de décisions. D'ailleurs, les hommes politiques d'aujourd'hui ne s'imposeraient plus à proprement parler comme des représentants du peuple, à savoir des individus dont la désignation « [...] a consisté en son principe à exprimer une identité ou à transmettre un mandat » (p. 21) ; aujourd'hui, ils seraient essentiellement des gouvernants dont « l'élection n'a fait que légitimer leur position institutionnelle et ne [leur] confère aucune qualité » (p. 21).

Toujours dans une perspective historique, Rosanvallon dresse le portrait des diverses figures politiques qui ont traversé le temps et présente leurs caractéristiques. Aujourd'hui, les gouvernants se distingueraient du prince-vertueux dont l'éducation morale était perçue comme primordiale, mais aussi de l'homme-peuple qui incarnait le pouvoir-un et personnifiait la nature de la démocratie ; de même ils sont loin de ressembler à l'homme politique par vocation - en référence à Max Weber (2003) - qui, lui, se distinguait par son total dévouement à la cause défendue, sa passion pour la tâche politique et son sens des responsabilités ; pour cette figure, seul le respect de l'intérêt général comptait.

De son côté, le monde contemporain regorgerait d'élus sans charisme, dépourvus de qualités et de vertus. La classe politique serait composée d'individus carriéristes, incompétents, cloitrés dans leur monde, dont l'unique but serait de représenter leurs propres partis. Ils ne seraient ni intègres ni dignes de confiance.

En vue d'enrayer cette " crise de la représentation » et ce défaut de démocratie qui consument nos sociétés, la mise en place d'un « bon gouvernement " s'imposerait alors. Celui-ci s'établirait par une redéfinition du rapport entre " gouvernés " et "gouvernants ". Les élus devraient y faire preuve de vertus, de qualités et d'un plus grand sens des responsabilités. Dans ce gouvernement dit "ouvert», une place de choix serait attribuée aux citoyens : la transparence et le « parler-vrai » seraient les principes sur lesquels se baserait ce type de pouvoir et viseraient à contrer l'opacité des décisions. Par l'adoption et la mise en place d'un bon gouvernement, la démocratie d'autorisation (à savoir celle qui accorde à un représentant par voie d'élection un « permis » de gouverner) se verrait prolonger pour devenir une démocratie d'exercice fondée sur trois éléments primordiaux : la lisibilité (consistant à rendre les décisions et actions politiques intelligibles pour les citoyens), la responsabilité (poussant les élus à tenir leurs engagements, à prendre des décisions qui s'inscriront dans le temps et non pas qui dureront uniquement le temps de l'élection...) et la réactivité (caractérisée par l'écoute et l'échange avec le peuple par la mise en place de débats publics par exemple). Par l'avènement d'une véritable démocratie d'exercice, les citoyens se verraient ainsi réattribuer un droit de regard sur le pouvoir, droit jusqu'ici usurpé par les gouvernants.

En conclusion, nous pouvons dire que cet ouvrage démontre de manière non équivoque que « le pouvoir, n'est pas une chose ; c'est une relation » (p. 22). Or, si en vue de l'établissement d'un bon gouvernement, le rapport gouvernés-gouvernants se doit d'être reconstruit politiquement, un retour des valeurs sociales et même éthiques dans la sphère de l'exécutif 
(telles que la confiance, l'intégrité, le parler-vrai, les qualités et les vertus) semble de même primordial en vue d'assurer la pérennité démocratique.

Cet ouvrage s'avère proposer une très bonne analyse des caractéristiques intrinsèques, pourtant trop souvent oubliées, de la démocratie. La force de ce livre réside dans le fait qu'il ne s'impose cependant pas comme une énième étude de ce régime politique : en effet, l'auteur semble avoir à cœur de retracer l'évolution historique de ce mode de gouvernement pour en comprendre non seulement les origines, mais aussi les raisons qui ont conduit au passage, depuis la Révolution, d'une démocratie d'autorisation et de représentation à un modèle présidentiel. Rosanvallon ne se contente pas de décrire les maux qui rongent la démocratie, mais en explique, exemples fournis à l'appui, le cheminement historique tout en proposant des alternatives pour la société future : ainsi, aujourd'hui, il nous faudrait mettre de côté la démocratie d'autorisation - par laquelle le citoyen permet à un élu de gouverner en lui attribuant son suffrage - à une démocratie d'exercice, où le citoyen " gouverne ", de concert, avec les représentants. Le rapport entre gouvernants et gouvernés se doit alors impérativement d'être redéfini : c'est d'ailleurs ce que préconise l'auteur qui tente à tout prix de remettre le peuple au centre de l'exercice politique.

En dépit des nombreuses qualités de cet ouvrage, il semblerait que le nombre de références historiques mobilisées ici, s'il représente une richesse non négligeable d'un point de vue scientifique et contribue inexorablement au renforcement des arguments de Rosanvallon, peut s'avérer trop important pour un public non averti. De plus, il est à regretter que l'auteur ait fait de son objectif premier de décrire l'évolution historique du mode démocratique au détriment d'une réflexion plus poussée sur le monde contemporain : en effet, l'auteur suggère de remplacer l'ancien modèle par une " démocratie de confiance » caractérisée par le " parlervrai » et l'intégrité. Cependant, dans cette partie pourtant essentielle, l'auteur n'évoque que trop brièvement les éléments nécessaires à l'application concrète de celle-ci dans la société actuelle.

Néanmoins, ces faiblesses ne remettent aucunement en cause la qualité de l'ouvrage qui s'inscrit dans le continuum de la pensée de Rosanvallon et dans lequel la démocratie est approchée en tant qu' « objet » d'analyse, sujet à une évolution permanente.

\section{Bibliographie}

DILMAÇ J.A. (2007), "La démocratie face à elle-même ", EspacesTemps.net. URL: www.espacestemps.net/en/articles/la-democratie-face-a-elle-meme

Rosanvallon P. (1992), Le sacre du citoyen. Histoire du suffrage universel en France, Paris, Gallimard (« Bibliothèque des Histoires »).

Rosanvallon P. (1998), Le peuple introuvable. Histoire de la représentation démocratique en France, Paris, Gallimard (" Bibliothèque des Histoires ").

Rosanvallon P. (2006), La contre démocratie : la politique à l'âge de la défiance, Paris, Seuil. 
Weber M. (2003), "La profession et la vocation de politique ", Le Savant et le Politique, Paris, La Découverte (" La Découverte Poche/Sciences humaines et sociales»). 